\title{
Activation of TLR-4 to produce tumour necrosis factor- $\alpha$ in neuropathic pain caused by paclitaxel
}

\author{
Z. Wu*, S. Wang*, I. Wu**, M. Mata, D.J. Fink \\ Department of Neurology, University of Michigan and VA Ann Arbor Healthcare System, USA
}

\author{
Correspondence \\ Zetang Wu \\ E-mail: zetang@umich.edu \\ Funding sources \\ These studies were supported by grants from \\ the Department of Veterans Affairs and the \\ $\mathrm{NIH}$ (NS038850 and BX002112) (to MM and \\ DJF). \\ Conflicts of interest \\ None declared. \\ * These two authors contributed equally to \\ this work. \\ ** Current address: \\ College of Art and Science, University of \\ Pennsylvania, Philadelphia, PA 19104 \\ Accepted for publication \\ 22 September 2014 \\ doi:10.1002/ejp.613
}

\begin{abstract}
Background: Neuropathic pain is a common complication of treatment with the anti-neoplastic drug paclitaxel. Animal studies suggest neuroinflammation and transient receptor potential channels TRPAl and TRPV4 are involved in the pathogenesis of pain in this condition. However, how neuroinflammation and TRPAl and TRPV4 are linked to cause pain in paclitaxel-treated animals is not known.

Methods: Paclitaxel-induced pain was modelled by IP injection of paclitaxel $(16 \mathrm{mg} / \mathrm{kg})$ once a week for 5 weeks. The role of toll-like receptor 4 (TLR-4) in tumour necrosis factor- $\alpha$ (TNF- $\alpha$ ) production and the effect of TNF- $\alpha$ on the expression of TRPAl and TRPV 4 were evaluated in vitro and in vivo. TNF- $\alpha$ signalling in dorsal root ganglion (DRG) was blocked by expressing soluble TNF receptor I (TNFsR) from a herpes simplex virus (HSV)-based vector (vTNFsR).

Results: Paclitaxel treatment increased the expression and release of TNF- $\alpha$ in satellite glial cells and increased the expression of TRPAl and TRPV4 in DRG neurons in animals. In vitro, paclitaxel enhanced the expression and release of TNF- $\alpha$ in enriched primary satellite glial cells, an effect that was blocked by an inhibitor of TLR-4. Direct application of TNF- $\alpha$ to primary DRG neurons in culture up-regulated the expression of TRPAl and TRPV4. In vivo, vector-mediated TNFsR release from DRG neurons reduced paclitaxel-induced up-regulation of TRPAl and TRPV4 expression and prevented paclitaxel-induced pain.
\end{abstract}

Conclusion: These results suggest that paclitaxel activation of TLR-4 to cause release of TNF- $\alpha$ from satellite glial cells increases the expression of TRPAl and TRPV4 in DRG neurons to cause neuropathic pain.

\section{Introduction}

Paclitaxel is a widely used anti-neoplastic agent and neuropathic pain is a common complication of the drug that not infrequently requires alteration in the treatment schedule or termination of the treatment (Dougherty et al., 2004). Paclitaxel-induced neuropathic pain is characterized by hypersensitivity to mechanical and cold stimuli that begins early after the initiation of treatment and may persist weeks or years after termination of the therapy (Dougherty et al., 2004).
Chronic pain and impairment in sensory nerve function are observed in cancer patients receiving paclitaxel treatment (Dougherty et al., 2004). But preclinical studies suggest that paclitaxel-induced pain is mechanistically independent of peripheral neuropathy: (1) paclitaxel induces pain at a dose that does not significantly alter sensory nerve function measured by electrophysiological testing (Flatters and Bennett, 2006); and (2) pain emerges long before deficits in sensory nerve function in patients, or in animals treated with a high-dose paclitaxel (Authier et al., 2000; Dougherty et al., 2004). 


\section{What's already known about this topic?}

- Transient receptor potential channels TRPAl and TRPV4 both contribute to paclitaxel-induced mechanical allodynia.

- TRPAl is responsible for paclitaxel-induced cold allodynia.

- Immunoactivation of sensory neurons plays a role in the pathogenesis of paclitaxel-induced neuropathic pain.

\section{What does this study add?}

- Paclitaxel induces tumour necrosis factor- $\alpha$ $(\mathrm{TNF}-\alpha)$ production in satellite glial cells of the dorsal root ganglion (DRG) through activation of toll-like receptor 4 .

- TNF- $\alpha$ mediates paclitaxel-induced neuropathic pain by up-regulating the expression of TRPAl and TRPV4 in DRG neurons.

There is indirect evidence for a role of neuroinflammation in the development of paclitaxel-induced pain. Pain-related behaviours in paclitaxel-treated animals are reduced by the anti-inflammatory agent thalidomide, the microglia activation inhibitor minocycline or by the anti-inflammatory cytokine IL10 (Ledeboer et al., 2007; Cata et al., 2008), and the production of tumour necrosis factor- $\alpha$ (TNF- $\alpha$ ), a key mediator of inflammation, is inhibited by these treatments (Ledeboer et al., 2007; Cata et al., 2008). The first aim of the current study was to directly evaluate the role of TNF- $\alpha$ in the pathogenesis of paclitaxel-induced pain.

In tissue culture, paclitaxel has been shown to be a ligand for the toll-like receptor 4 (TLR-4), causing LPS-like activation of the TLR-4 receptor to induce production of pro-inflammatory cytokines including TNF- $\alpha$ (Byrd-Leifer et al., 2001). In addition to its role in innate immunity, TLR-4 has been shown to participate in the pathogenesis of neuropathic pain in other models of nerve injury (Bettoni et al., 2008; Wu et al., 2010). TLR-4 is constitutively expressed in both satellite glial cells and neurons in dorsal root ganglia (DRG) (Barajon et al., 2009) and paclitaxel accumulates in DRG after administration (Cavaletti et al., 2000). The second aim of this study was to investigate how paclitaxel induces TNF- $\alpha$ expression by examining the role of TLR- 4 activation in TNF- $\alpha$ expression and cleavage.

Both TRPAl and TRPV4 contribute to paclitaxelinduced mechanical allodynia (Alessandri-Haber et al., 2004) while TRPAl is critical for paclitaxelinduced cold allodynia (Chen et al., 2011; Materazzi et al., 2012). The third aim of the current study was to examine the effect of blocking TNF- $\alpha$ signalling on the expression of these two cation channels in paclitaxeltreated animals in order to understand how TNF- $\alpha$ signalling might lead to paclitaxel-induced pain.

\section{Materials and methods}

\subsection{Experimental animals}

Male Sprague-Dawley rats weighing 250-300 g were used in these experiments. Animals were housed 2 per cage for 1 week before use under a light/dark (12:12 h) cycle and provided with food and water ad libitum. All procedures were performed using protocols approved by the Institutional Animal Care and Use Committee.

\subsection{Reagents}

Recombinant rat TNF- $\alpha$ (R\&D Systems, Minneapolis, MN, USA), which contains the soluble extracellular portion (sTNF- $\alpha$ ) of rat TNF- $\alpha$ (amino acids 80-235) (Mohan et al., 2002), was used in vitro studies. TLR-4 inhibitor TAK-242 (InvivoGen, San Diego, CA, USA) was suspended in DMSO (Thermo Scientific, Rockford, IL, USA) to make $3 \mathrm{mM}$ stock and diluted to a working concentration $(3 \mu \mathrm{M})$ in culture medium (Kawamoto et al., 2008).

\subsection{Paclitaxel administration}

Paclitaxel (6 mg/mL in 50:50 cremophor EL/ethanol) was purchased from Bristol-Myers Squibb (Princeton, NJ, USA) and diluted to $2 \mathrm{mg} / \mathrm{mL}$ with saline just before use. Animals were injected with paclitaxel (16 mg/kg, IP) once a week for 5 weeks to model paclitaxel-induced painful peripheral neuropathy (Authier et al., 2000). Cremophor EL/ethanol (50:50) diluted threefold with saline was used as the vehicle control.

\subsection{Vector injection}

The replication-defective herpes simplex virus (HSV)-based vector (vTNFsR), which expresses soluble TNF receptor I (TNFsR) under the regulatory control of the HSV ICP0 immediate-early promoter (Peng et al., 2006; Hao et al., 2007), was used to block TNF- $\alpha$ signalling in animals. VTNFsR or control vector expressing green fluorescent protein constructed in the same HSV backbone $\left(3 \times 10^{8}\right.$ plaque forming units in $30 \mu \mathrm{L}$ PBS) was injected subcutaneously into the plantar surface of both hind paws twice. The vectors were injected 3 days before the first dose of paclitaxel, and a repeat injection was performed 2 weeks later.

\subsection{TNFsR release from DRG neurons of animals}

Three days after vector injection, L4-6 DRGs from both sides of each animal were dissected, prepared as previously 
described (Wu et al., 2005), seeded into wells of a 12-well plate and cultured in Neurobasal medium supplemented with B27, Glutamax I, Albumax, Pstrep and 7.0S nerve growth factor (Wu et al., 2011). Medium was collected for TNFsR enzyme-linked immunosorbent assay (ELISA) after 24-h culture.

\subsection{Primary cell culture}

E17 primary DRG neurons were isolated and cultured as previously described (Wu et al., 2011). DRG neurons at 7 days in vitro were used in the studies. Enriched primary satellite glial cells were prepared using a method described by Arora et al. (2007) with minor modifications. The actual procedures used for preparing enriched primary satellite glial cells are given in Supporting Information Methods S1.

\subsection{Behavioural testing}

Mechanical and cold allodynia was measured using dynamic plantar aesthesiometer and a method described by Chattopadhyay et al. (2008) with slight modifications. The detailed information for the testing is given in supporting Information Methods S2 and S3.

\subsection{Quantitative PCR}

Total RNA isolation and quantitative PCR (qPCR) were conducted as previously described (Wu et al., 2011). Primers used for qPCR are given in Supporting Information Table S1. mRNA levels were normalized to $\beta$-actin. mRNA amounts in treatment groups were compared with the respective control groups with the control mRNA level set at 1 .

\subsection{Biochemical analysis}

Protein levels were analysed by Western blotting. Soluble TNF- $\alpha$ and TNFsR in medium, and soluble TNF- $\alpha$ in DRG lysates were determined by ELISA. TNF- $\alpha$ converting enzyme (TACE) activity was evaluated as previously described (Wu et al., 2011). Cellular source of protein expression in DRG of animals was determined by immunohistochemistry. In situ hybridization of TNF- $\alpha$ mRNA was carried out using the DIG DNA Labeling and Detection Kits from Roche (Indianapolis, IN, USA) according to the manufacturer's instructions. The detailed procedures for all the assays and the information for the preparation of digoxigenin-labelled TNF- $\alpha$ RNA for in situ hybridization are described in Supporting Information Methods S4-S9.

\subsection{Statistical analysis}

In vitro experiments were repeated three times and the statistical significance of the difference between treatments was determined by Student's $t$-test. The statistical significance of the difference between groups in animal studies was deter- mined by analysis of variance (ANOVA) and corrected using Bonferroni's method. Results are expressed as mean \pm SEM.

\section{Results}

\subsection{Paclitaxel increases TNF- $\alpha$ mRNA and enhances TACE activity resulting in TNF- $\alpha$ cleavage in DRG}

In order to explore the role of TNF- $\alpha$ in the pathogenesis of paclitaxel-induced neuropathic pain, we examined the effect of paclitaxel administration on the expression and cleavage of TNF- $\alpha$ in the DRG of animals. We modelled paclitaxel-induced painful peripheral neuropathy by injecting rats with paclitaxel ( $16 \mathrm{mg} / \mathrm{kg}$, IP) once a week for 5 weeks (Authier et al., 2000). Animals treated with paclitaxel developed mechanical allodynia and cold allodynia that were apparent at 2 weeks and persisted through 5 weeks of paclitaxel treatment (Fig. 1) consistent with previous
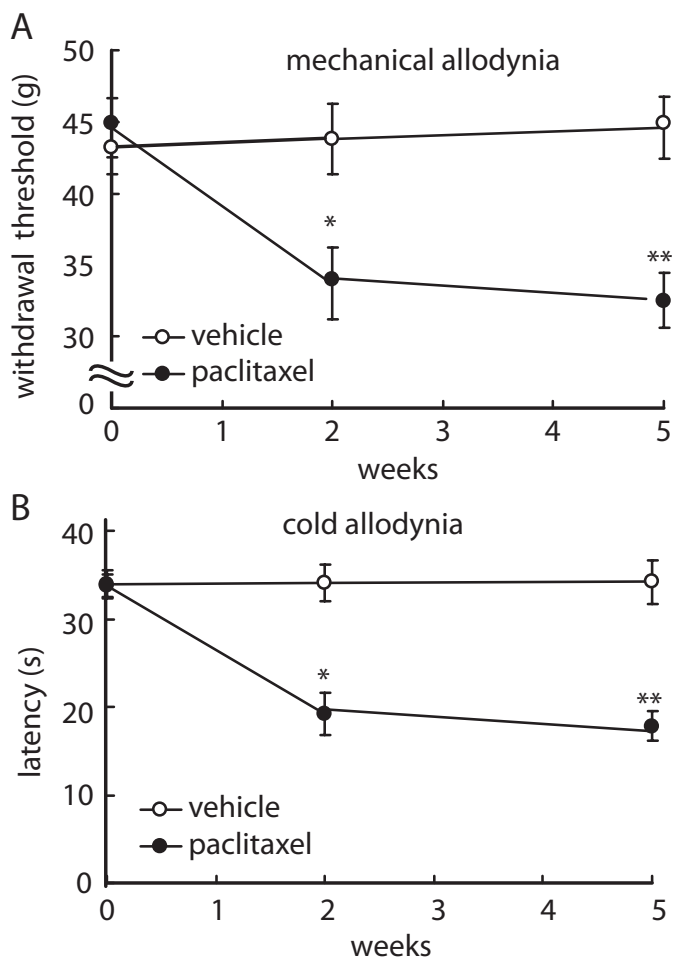

Figure 1 Paclitaxel-induced mechanical and cold allodynia. Paclitaxelinduced neuropathic pain was modelled by repeated injection of paclitaxel as described in the Materials and methods section. Mechanical and cold allodynia was evaluated 2 and 5 weeks after the initiation of paclitaxel treatment. Pain-related behaviours in paclitaxel-treated animals were compared with those observed in vehicle-treated animals at each time point. (A) Mechanical allodynia. (B) Cold allodynia. $N=10$ animals per group. ${ }^{*} p \leq 0.05 ;{ }^{*} p \leq 0.01$. 


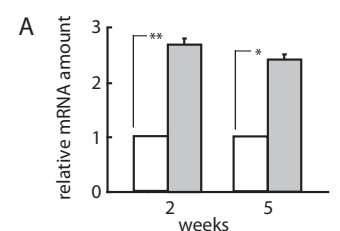

$\square$ vehicle $\square$ paclitaxel

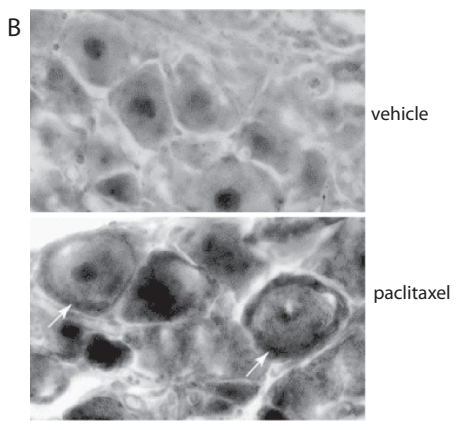

D

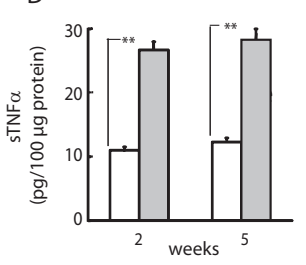

$\square$ vehicle $\square$ paclitaxel

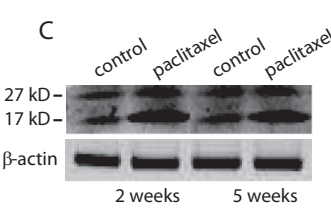

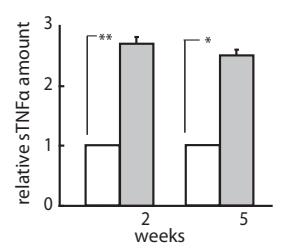

$\square$ vehicle $\square$ paclitaxel

E

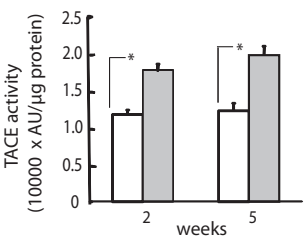

$\square$ vehicle $\square$ paclitaxel
Figure 2 Paclitaxel-induced expression and cleavage of TNF- $\alpha$ in satellite glial cells in DRG. Paclitaxel-induced neuropathic pain was modelled by repeated injection of paclitaxel. TNF- $\alpha$ expression and TACE activity in DRG were determined 2 and 5 weeks after the initiation of paclitaxel treatment. (A) TNF- $\alpha$ qPCR. (B) TNF- $\alpha$ in situ hybridization (arrows: satellite glial cells; 5 weeks of paclitaxel). (C) Western blot and quantification of STNF- $\alpha$ (17 kD band). (D) STNF- $\alpha$ ELISA. (E) TACE activity. Comparison between treatment and control groups was made at each time point. $n=6$ animals per group for Western blot, ELISA and qPCR determinations. ${ }^{*} p \leq 0.05 ; * x p 0.01$. reports (Authier et al., 2000). Animals receiving paclitaxel showed increased TNF- $\alpha$ mRNA in DRG at 2 and 5 weeks after the initiation of paclitaxel administration compared with vehicle-treated animals (Fig. 2A). To identify the cellular source of TNF- $\alpha$ mRNA expression, we performed in situ TNF- $\alpha$ mRNA hybridization of DRG sections after 5 weeks of paclitaxel treatment. TNF- $\alpha$ mRNA was localized predominantly in satellite glial cells surrounding DRG neurons in paclitaxeltreated animals (Fig. 2B). The increase in TNF- $\alpha$ mRNA was reflected by an increase in sTNF- $\alpha$ without accumulation of mTNF- $\alpha$ (Fig. 2C), suggesting that most of the mTNF- $\alpha$ produced was cleaved into sTNF- $\alpha$. The increase in sTNF- $\alpha$ was confirmed by ELISA (Fig. 2D), and correlated with an increase in TACE activity in the DRG of paclitaxel-treated animals (Fig. 2E).

\subsection{Paclitaxel increases TRPA1 and TRPV4 expression in DRG neurons in animals}

Previous work using knockout animals or channel inhibitors indicates that TRPAl is critical for the development of paclitaxel-induced cold allodynia (Chen et al., 2011; Materazzi et al., 2012), and both TRPAl and TRPV4 contribute to paclitaxel-induced mechanical allodynia (Alessandri-Haber et al., 2004). We found a significant increase in the expression of TRPAl and TRPV4 at both mRNA and protein levels measured by qPCR (Fig. 3A) and by Western blot (Fig. 3B) in paclitaxel-treated animals after 2 and 5 weeks of treatment and confirmed by immunostaining of DRG sections prepared from animals after 5 weeks of treatment, which demonstrated TRPAl localization in a portion of medium and small neurons and TRPV4 expression in a portion of medium and large neurons of DRG, respectively (Fig. 3C). Pre-incubation of the antigen with the antibody prior to incubation of sections with the primary antibody resulted in no fluorescent signals in sections prepared from both paclitaxel and vehicle-treated animals (data not shown) suggesting the specificity of the TRPAl and TRPV4 antibodies.

\subsection{Inhibition of TNF- $\alpha$ signalling in vivo blocks paclitaxel-induced increase in the expression of TRPA1 and TRPV4 in DRG, and prevents paclitaxel-induced pain}

TNFsR is the truncated form of human p55 TNFR I containing amino acids $1-211$ of p55 TNFR I. TNFsR competes with TNFR to bind to TNF- $\alpha$, thus blocking TNF- $\alpha$ signalling (Engelmann et al., 1990). We constructed an HSV vector vTNFsR that expresses TNFsR and in vivo studies indicate that TNFsR is released into the dorsal horn of spinal cord from primary afferent terminals and blocks TNF- $\alpha$ signalling in spinal dorsal horn (Peng et al., 2006; Hao et al., 2007). In order for exploiting that vector to block TNF- $\alpha$ signalling in DRG, we tested the release of TNFsR from soma after expressed from DRG neurons from the vector. Animals were injected with either vTNFsR or control vector expressing green fluorescent protein (GFP) at both hind paws. L4-6 DRGs from both hind legs were dissected 3 days after vector inoculation, DRG neurons partially dissociated and cultured. Medium was collected after $24 \mathrm{~h}$ in vitro and TNFsR in the medium was measured by ELISA. DRG neurons from animals trans- 
A

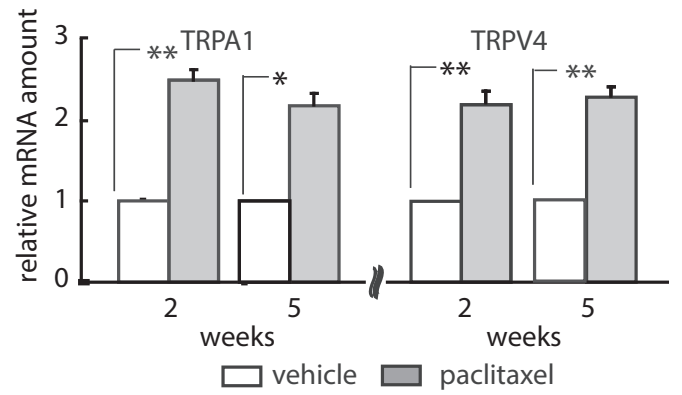

B
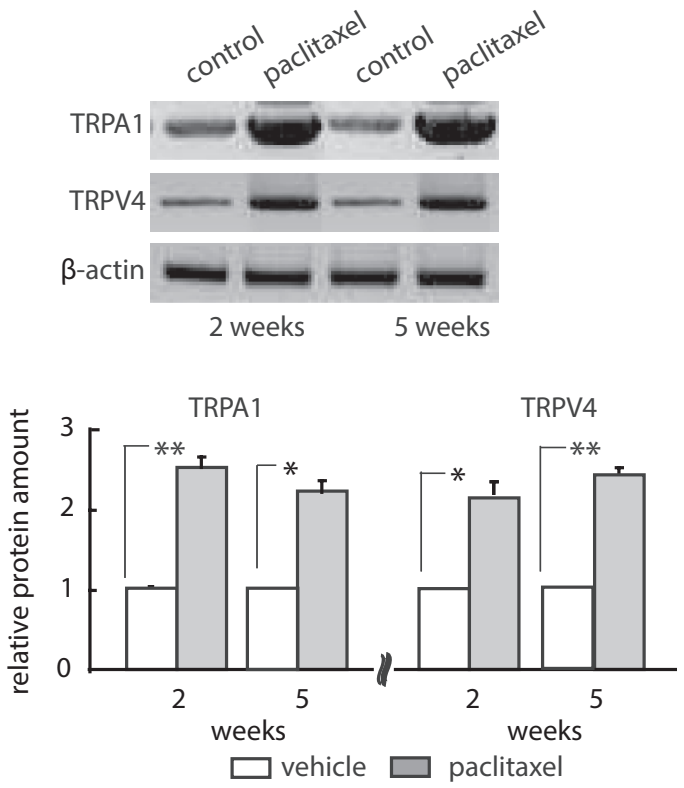

C vehicle paclitaxel
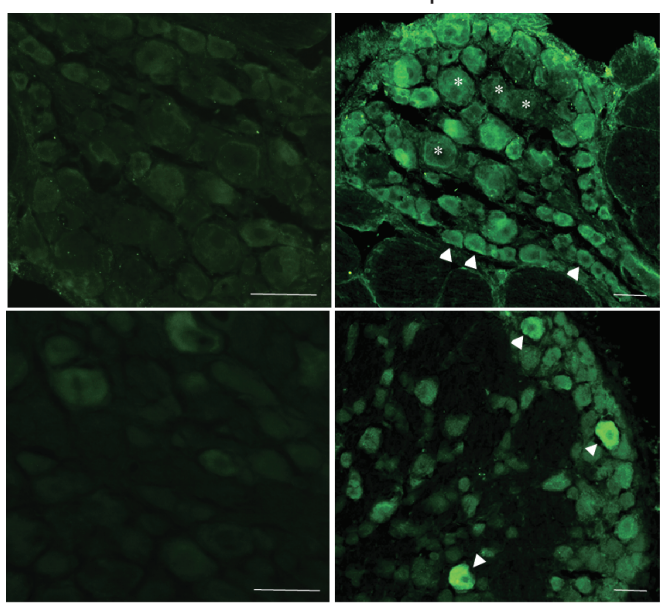

fected with vTNFsE released into the medium $1.8 \pm 0.09 \mathrm{ng} / \mathrm{mL}$ of TNFsR corresponding to $150 \mathrm{pg} /$ DRG neuron per day while TNFsR was below the detection in the culture medium of DRG neurons
Figure 3 Paclitaxel increased the expression of TRPA1 and TRPV4 in DRG in vivo. Paclitaxel-induced neuropathic pain was modelled by repeated injection of paclitaxel. TRPA1 and TRPV 4 expression in DRG was evaluated 2 and 5 weeks after the initiation of paclitaxel treatment. Comparison between treatment and control groups was made at each time point. (A) TRPA 1 and TRPV4 qPCR. (B) TRPA1 and TRPV4 Western blot: Western blot (upper) and quantification (lower). (C) Immunostaining for TRPA1 and TRPV4 in DRG (5 weeks of paclitaxel). Arrowhead: medium and small neurons for TRPA1; medium and large neurons for TRPV4. Star: large neurons for TRPA1. Bar $=50 \mu \mathrm{m} . n=4$ animals per group for immunohistochemistry; $n=6$ animals per group for Western blot and qPCR. ${ }^{*} p \leq 0.05 ;{ }^{* *} p \leq 0.01$

obtained from control vector-infected animals. The results confirm that TNFsR is expressed and released from DRG neurons transduced by vTNFsR in vivo. To examine the role of TNF- $\alpha$ produced in DRG in the pathogenesis of paclitaxel-induced pain, we tested the effect of blocking TNF- $\alpha$ signalling on the expression of TRPAl and TRPV4 in DRG and on the development of neuropathic pain in paclitaxel-treated animals. vTNFsR was injected subcutaneously into both hind foot paws 3 days before the start of paclitaxel administration and again 2 weeks later to ensure a robust expression of TNFsR throughout the 5 weeks of paclitaxel treatment. Animals injected with vTNFsR but not animals injected with the control vector expressing GFP showed a substantial reduction in pain-related behaviours at 2 and 5 weeks of paclitaxel treatment (Fig. 4A). The reduction in pain-related behaviours correlated with a blunting of the increase in the expression of TRPAl and TRPV4 mRNA (Fig. 4B) and protein (Fig. 4C) in the DRG of paclitaxel-treated animals inoculated with vTNFsR, an effect that was not seen in paclitaxel-treated animals inoculated with control vector.

\subsection{Paclitaxel enhances the expression and release of TNF- $\alpha$ from primary satellite glial cells in a TLR-4-dependent manner}

Because we found that paclitaxel induces the expression and cleavage of TNF- $\alpha$ in satellite glial cells in animals and activation of TLR- 4 is known to enhance TNF- $\alpha$ expression and cleavage (Byrd-Leifer et al., 2001), we examined the effect of paclitaxel on the expression and release of TNF- $\alpha$ in enriched satellite glial cells in vitro. Enriched satellite glial cells were prepared from DRG dissected from 17-day rat embryos (Arora et al., 2007) and characterized by co-expression of $S 100 \beta$ and RAMPI (Eftekhari et al., 2010). TLR-4 protein was expressed in those cells and the level of that expression was not changed by 
A
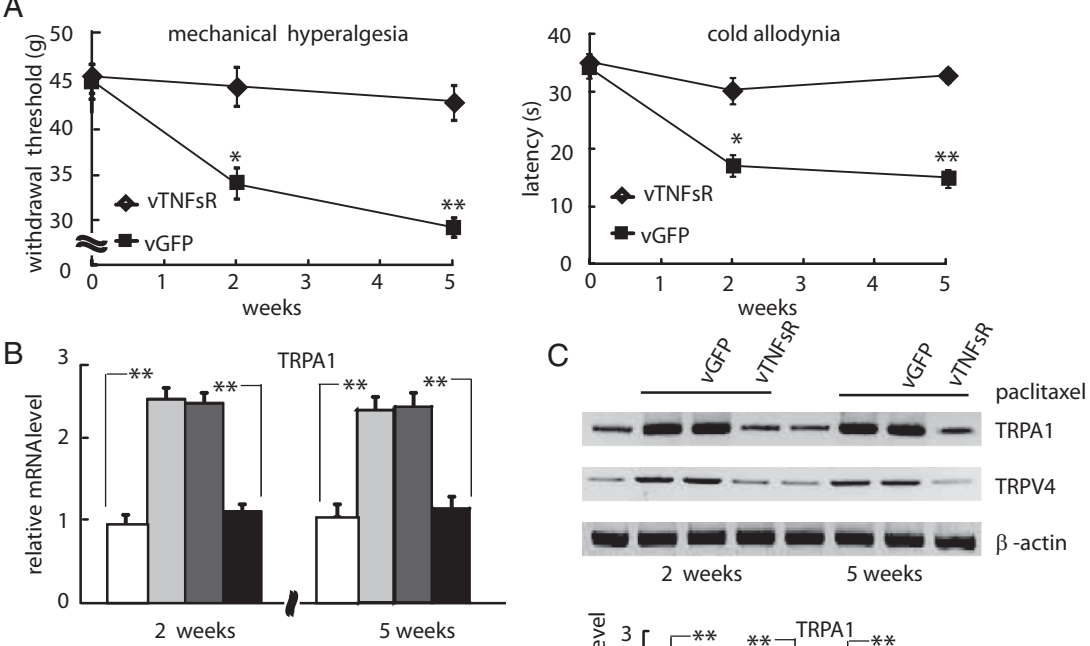

Figure 4 TNFsR expressed and released from an HSV vector in vivo prevented pain-related behaviours and blocked the increases in TRPA1 and TRPV4. Paclitaxel-induced neuropathic pain was modelled by repeated injection of paclitaxel and TNFsR expressing vector VTNFSR injected 3 days before the initiation of paclitaxel treatment and 2 weeks later. Pain-related behaviours in animals and TRPA 1 and TRPV4 expression in DRG were evaluated 2 and 5 weeks after the initiation of paclitaxel treatment. Comparison between vTNFsR and control vector-treated animals was made at each time point. (A) Mechanical (left) and cold allodynia (right). (B) TRPA1 and TRPV4 qPCR. (C) TRPA1 and TRPV4 Western blot (upper) and quantification (middle and lower). $n=10$ animals per group for behavioural testing; $n=4$ animals per group for immunohistochemistry; $n=6$ animals per group for mRNA and protein analysis. ${ }^{\star} p \leq 0.05 ;{ }^{*} p \leq 0.01$.

exposure to paclitaxel (Fig. 5A). However, exposure to paclitaxel increased TNF- $\alpha$ mRNA (Fig. 5B) and enhanced TNF- $\alpha$ release from those cells (Fig. $5 \mathrm{C}$ ) in a dose-dependent manner. Paclitaxel-induced enhancement in TNF- $\alpha$ mRNA expression and TNF- $\alpha$ release from those cells was blocked by co-treatment with the TLR-4 antagonist TAK-242 (3 $\mu \mathrm{mol} / \mathrm{L})$ (Kawamoto et al., 2008) (Fig. 5B and C).

\subsection{Exposure to recombinant TNF- $\alpha$ increases the expression of TRPA1 and TRPV4 in cultured primary DRG neurons}

To determine whether sTNF- $\alpha$ directly acts on DRG neurons to increase the expression of TRPAl and TRPV4, we examined the effect of recombinant TNF- $\alpha$ on the expression of TRPAl and TRPV4 in primary DRG neurons in culture. Exposure to recombinant TNF- $\alpha$ increased TRPAl and TRPV4 mRNA (Fig. 6A) and protein (Fig. 6B) in primary DRG neurons in a dose-dependent manner with the maximal effect observed in cells exposed to $15 \mathrm{ng} / \mathrm{mL}$ of recombinant TNF- $\alpha$.

\subsection{Paclitaxel does not increase TNF- $\alpha$ release from primary DRG neurons in vitro and has no effect on the expression of TRPA1 and TRPV4 proteins}

TLR-4 is also expressed in DRG neurons (Barajon et al., 2009). To examine whether activation of TLR-4 in neurons contributes to paclitaxel-induced neuropathic pain, we tested the effect of paclitaxel on the release of TNF- $\alpha$ and on the protein expression of TRPAl and TRPV4 in cultured primary DRG neurons. Paclitaxel treatment had no effect on the release of TNF- $\alpha$ compared with control (Supporting Information Fig. Sla), and no significant difference in TRPAl and TRPV4 protein levels was observed in paclitaxeltreated cells compared with control cells in the presence and the absence of TLR-4 antagonist TAK-242 (Supporting Information Fig. S1b).

\section{Discussion}

Our results suggest that paclitaxel, acting through TLR-4, enhances the expression and cleavage of TNF- $\alpha$ in satellite glial cells in DRG, and that release of 
A

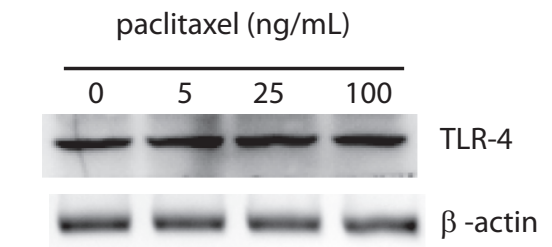

B

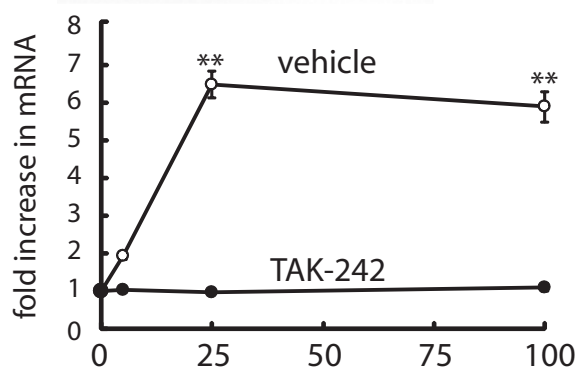

C

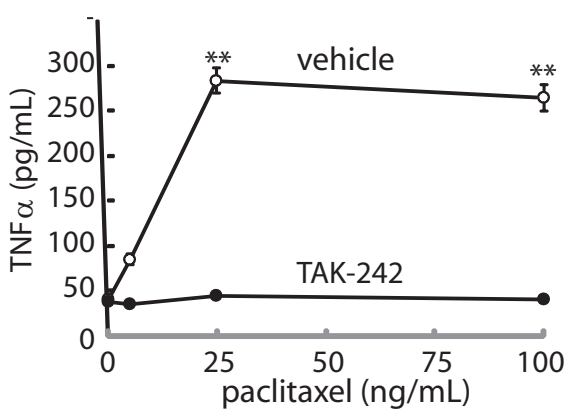

Figure 5 Paclitaxel increased the expression and release of TNF- $\alpha$ in an enriched satellite glial cell culture in vitro. Enriched satellite glial cell culture was treated with paclitaxel of different concentrations in the presence or absence of TLR- 4 inhibitor TAK-242. TLR-4 protein levels, TNF- $\alpha$ mRNA expression and release were determined after $24 \mathrm{~h}$ of culture. Comparison was made between the inhibitor group and the vehicle group at each paclitaxel dose. (A) Western blot of TLR-4. (B) TNF- $\alpha$ qPCR. (C) TNF- $\alpha$ ELISA. ${ }^{* *} p \leq 0.01$.

sTNF- $\alpha$ from those cells increases expression of TRPAl and TRPV4 in DRG neurons resulting in a phenotypic change that causes neuropathic pain (Fig. 7).

Previous studies suggest the involvement of TNF- $\alpha$ in the development of paclitaxel-induced pain (Ledeboer et al., 2007; Cata et al., 2008). In the studies we reported here, we found paclitaxel increased the expression of TNF- $\alpha$ in DRG and that specifically blocking TNF- $\alpha$ signalling by expressing TNFsR to sequester TNF- $\alpha$ abrogated paclitaxel-induced pain. Expression and release of TNFsR from DRG transduced by vector vTNFsR in vivo does not alter painrelated behaviours in naive animals (Hao et al., 2007). These results confirm that TNF- $\alpha$ signalling is required for the facilitation of paclitaxel-induced pain.

We found that the increased expression of TNF- $\alpha$ was accompanied by enhanced activity of the TACE in paclitaxel-treated animals leading to an increase in
TNF- $\alpha$ cleavage and release. These results indicate sTNF- $\alpha$ is the functional form of TNF- $\alpha$ that signals to cause pain in paclitaxel-treated animals. This is in contrast to the observation in neuropathic pain resulting from physical nerve injury where up-regulated TNF- $\alpha$ mRNA is reflected by a significant increase in MTNF- $\alpha$ protein in DRG while no change in cleavage or increase in release of TNF- $\alpha$ was seen (Schafers et al., 2003; Sacerdote et al., 2008).

In situ hybridization showed that increased TNF- $\alpha$ expression occurred in satellite glial cells in paclitaxeltreated animals. Consistent with this in vivo observation, we found paclitaxel increased the expression and release of TNF- $\alpha$ from enriched satellite glial cells in culture, an effect that was blocked by TLR-4 inhibitor. In contrast, paclitaxel had no effect on the expression and release of TNF- $\alpha$ in primary DRG neurons in culture. Although TLR-4 is constitutively expressed in satellite glial cells and DRG neurons in animals (Barajon et al., 2009), our results suggest that TLR-4

A

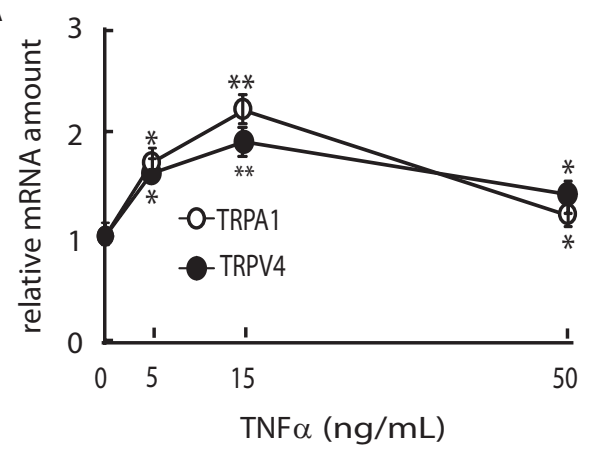

B

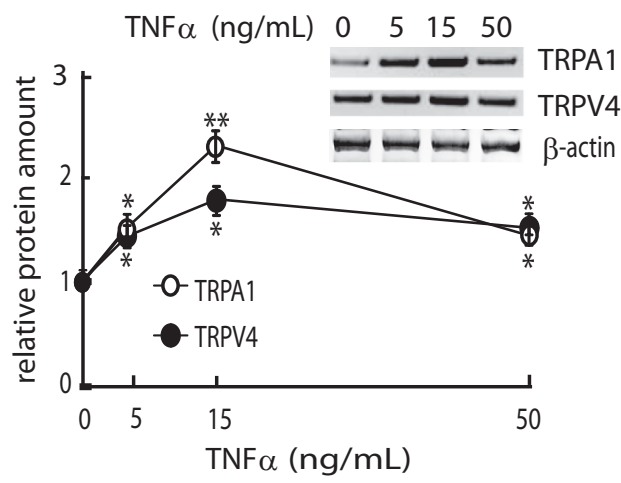

Figure 6 Recombinant TNF- $\alpha$ induced the expression of TRPA1 and TRPV4 in primary DRG neurons in vitro. Primary DRG neurons in culture were treated with recombinant TNF- $\alpha$ of different concentrations. TRPA1 and TRPV4 expression in cells was evaluated $24 \mathrm{~h}$ after treatment. Comparison was performed between treatment and control (no treatment). (A) TRPA 1 and TRPV4 qPCR. (B) TRPA1 and TRPV4 Western blot (inset) and quantification ${ }^{*} p \leq 0.05 ;{ }^{*} p \leq 0.01$. 


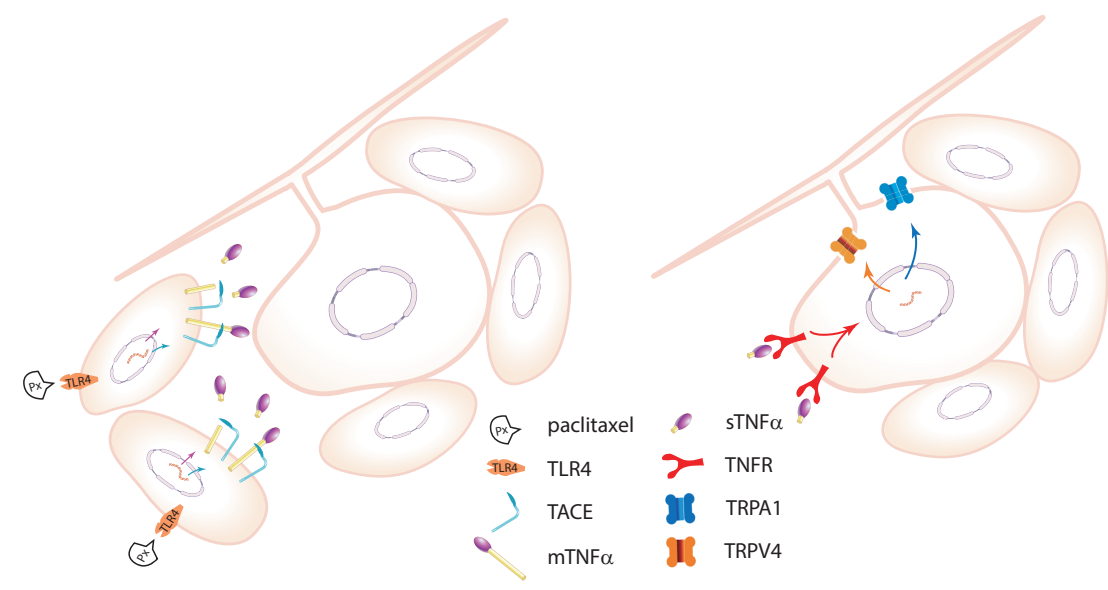

Figure 7 Schematic representation of the proposed signalling pathway mediating paclitaxelinduced neuropathic pain. Paclitaxel binding to TLR-4 in satellite glial cells induces the expression of TNF- $\alpha$ and increases TACE activity leading to release of sTNF- $\alpha$ (left), sTNF- $\alpha$ binding to the TNF receptor enhances the expression of TRPA1 and TRPV4 in DRG neurons to cause pain (right). activation in satellite glial cells not in DRG neurons is responsible for the elevated expression and cleavage of TNF- $\alpha$ in paclitaxel-treated animals.

In order to understand how STNF- $\alpha$ signalling leads to cause pain in paclitaxel-treated animals, we tested the effect of recombinant TNF- $\alpha$ on the expression of TRP channel proteins TRPAl and TRPV4 in primary DRG neurons in culture. Genetic and pharmacological studies indicate the roles of TRPAl and TRPV4 in the development of paclitaxel-induced pain (AlessandriHaber et al., 2004; Materazzi et al., 2012). Recombinant TNF- $\alpha$ enhanced the expression of TRPAl and TRPV4 in primary DRG neurons in culture. In agreement with the in vitro observations, we found in animals that blocking TNF- $\alpha$ signalling through expression of TNFsR in DRG from an HSV vector blunted the increase in the expression of TRPAl and TRPV4 in DRG and abrogated paclitaxel-induced pain. Taken together, our results suggest that STNF- $\alpha$ produced in satellite glial cells gets involved in the pathogenesis of paclitaxel-induced pain by up-regulating the expression of channel proteins TRPAl and TRPV4 in DRG neurons.

TACE is the major enzyme responsible for the cleavage of TNF- $\alpha$. We found in our previous studies that activation of TLR- 4 by LPS up-regulates TACE expression and activity, thus resulting in cleavage and release of TNF- $\alpha$ (Zhou et al., 2010). In the current study, we found paclitaxel mimicked the activity of LPS to enhance the expression and release of TNF- $\alpha$ via activation of TLR-4 in primary satellite glial cell culture and in vivo studies indicated paclitaxel increased TACE activity in DRG. These results point out enhanced TACE activity observed in paclitaxel-treated animals might result from direct activation of TLR-4 in satellite glial cells by paclitaxel.

Paclitaxel inhibits axonal growth in DRG neurons in a dose-dependent manner by TLR-4-dependent and independent mechanisms (Ustinova et al., 2013). However, it is unlikely that these neurotoxic effects of paclitaxel on DRG neurons are related to the development of pain because paclitaxel-induced pain appears to be temporally independent of the development of peripheral sensory neuropathy (Authier et al., 2000; Dougherty et al., 2004), and we found no evidence of a direct effect of paclitaxel on DRG expression of TRP channels.

Recent studies show that paclitaxel may alter the phenotype of neurons and glia through mechanisms of action that differ depending on concentration. In vitro, paclitaxel (10 nM) enhances TRPVl agonist (capsaicin)-evoked release of CGRP from DRG neurons, while higher dose (300 nM) paclitaxel inhibits capsaicin-evoked release of CGRP (Pittman et al., 2014). Whether paclitaxel alters channel function of TRPAl and TRPV4 in a similar fashion is not known. Another study (Li et al., 2014) reported that low-dose paclitaxel ( $1-2 \mathrm{mg} / \mathrm{kg}$ per dose) in vivo up-regulates expression of TLR-4 in DRG neurons and in astrocytes of the dorsal horn of spinal cord. We did not observe any change in TLR-4 expression in animals treated with high dose (16 mg/kg per dose; data not shown). However, the observation by Li et al. (2014) that intrathecal administration of a TLR-4 antagonist (LPSRS) reverses paclitaxel-induced pain is consistent with the TLR-4-dependent mechanism of paclitaxelinduced pain that we have proposed.

Several inflammatory cytokines including IL1 $\beta$ are implicated in the pathogenesis of low-dose paclitaxelinduced neuropathic pain in animals (Ledeboer et al., 2007). We found that expression and release of TNFsR from DRG neurons prevents high-dose paclitaxelinduced pain in the current study, but the experiments performed in this study do not rule out the possibility that other inflammatory cytokines might be involved in the pathogenesis of pain, as we and others have 
demonstrated that blocking TNF- $\alpha$ signalling by TNF- $\alpha$ neutralizing antibody or by TNFsR expressed from an HSV vector inhibits the expression not only of TNF- $\alpha$ but also of other inflammatory cytokines (Hao et al., 2007; Yamakawa et al., 2011).

\subsection{Conclusion}

In summary, the results presented herein suggest paclitaxel activation of TLR-4 to cause release of TNF- $\alpha$ from satellite glial cells increases expression of TRPAl and TRPV4 in neurons of the DRG to cause neuropathic pain and that targeting either TLR-4, TNF- $\alpha$ signalling or transient receptor potential channel proteins may represent the strategies for developing therapies for ameliorating neuropathic pain in patients receiving paclitaxel therapy.

\section{Author contributions}

Z.W.: Designed the experiments, conducted the research and wrote the manuscript. S.W.: Conducted the research and reviewed/edited the manuscript. I.W.: Participated in the research and reviewed/edited the manuscript. M.M.: Designed the experiments and reviewed/edited the manuscript. D.J.F.: Designed the experiments and wrote/edited the manuscript.

\section{Acknowledgement}

We gratefully acknowledge Mr Vikram Thakur in preparation of the vectors used in this study.

\section{References}

Alessandri-Haber, N., Dina, O.A., Yeh, J.J., Parada, C.A., Reichling, D.B., Levine, J.D. (2004). Transient receptor potential vanilloid 4 is essential in chemotherapy-induced neuropathic pain in the rat. $J$ Neurosci 24, 4444-4452.

Arora, D.K., Cosgrave, A.S., Howard, M.R., Bubb, V., Quinn, J.P., Thippeswamy, T. (2007). Evidence of postnatal neurogenesis in dorsal root ganglion: Role of nitric oxide and neuronal restrictive silencer transcription factor. $J$ Mol Neurosci 32, 97-107.

Authier, N., Gillet, J.P., Fialip, J., Eschalier, A., Coudore, F. (2000). Description of a short-term Taxol-induced nociceptive neuropathy in rats. Brain Res 887, 239-249.

Barajon, I., Serrao, G., Arnaboldi, F., Opizzi, E., Ripamonti, G., Balsari, A., Rumio, C. (2009). Toll-like receptors 3, 4, and 7 are expressed in the enteric nervous system and dorsal root ganglia. J Histochem Cytochem 57, 1013-1023.

Bettoni, I., Comelli, F., Rossini, C., Granucci, F., Giagnoni, G., Peri, F., Costa, B. (2008). Glial TLR4 receptor as new target to treat neuropathic pain: Efficacy of a new receptor antagonist in a model of peripheral nerve injury in mice. Glia 56, 1312-1319.

Byrd-Leifer, C.A., Block, E.F., Takeda, K., Akira, S., Ding, A. (2001). The role of MyD88 and TLR4 in the LPS-mimetic activity of Taxol. Eur J Immunol 31, 2448-2457.
Cata, J.P., Weng, H.R., Dougherty, P.M. (2008). The effects of thalidomide and minocycline on taxol-induced hyperalgesia in rats. Brain Res 1229 , $100-110$.

Cavaletti, G., Cavalletti, E., Oggioni, N., Sottani, C., Minoia, C., D’Incalci, M., Zucchetti, M., Marmiroli, P., Tredici, G. (2000). Distribution of paclitaxel within the nervous system of the rat after repeated intravenous administration. Neurotoxicology 21, 389-393.

Chattopadhyay, M., Mata, M., Fink, D.J. (2008). Continuous delta-opioid receptor activation reduces neuronal voltage-gated sodium channel (NaV1.7) levels through activation of protein kinase $\mathrm{C}$ in painful diabetic neuropathy. J Neurosci 28, 6652-6658.

Chen, Y., Yang, C., Wang, Z.J. (2011). Proteinase-activated receptor 2 sensitizes transient receptor potential vanilloid 1 , transient receptor potential vanilloid 4, and transient receptor potential ankyrin 1 in paclitaxel-induced neuropathic pain. Neuroscience 193, 440-451.

Dougherty, P.M., Cata, J.P., Cordella, J.V., Burton, A., Weng, H.R. (2004). Taxol-induced sensory disturbance is characterized by preferential impairment of myelinated fiber function in cancer patients. Pain 109, 132-142.

Eftekhari, S., Salvatore, C.A., Calamari, A., Kane, S.A., Tajti, J., Edvinsson, L. (2010). Differential distribution of calcitonin gene-related peptide and its receptor components in the human trigeminal ganglion. Neuroscience 169, 683-696.

Engelmann, H., Novick, D., Wallach, D. (1990). Two tumor necrosis factor-binding proteins purified from human urine. Evidence for immunological cross-reactivity with cell surface tumor necrosis factor receptors. J Biol Chem 265, 1531-1536.

Flatters, S.J., Bennett, G.J. (2006). Studies of peripheral sensory nerves in paclitaxel-induced painful peripheral neuropathy: Evidence for mitochondrial dysfunction. Pain 122, 245-257.

Hao, S., Mata, M., Glorioso, J.C., Fink, D.J. (2007). Gene transfer to interfere with TNFalpha signaling in neuropathic pain. Gene Ther 14, 1010-1016.

Kawamoto, T., Ii, M., Kitazaki, T., Iizawa, Y., Kimura, H. (2008). TAK-242 selectively suppresses Toll-like receptor 4 -signaling mediated by the intracellular domain. Eur J Pharmacol 584, 40-48.

Ledeboer, A., Jekich, B.M., Sloane, E.M., Mahoney, J.H., Langer, S.J., Milligan, E.D., Martin, D., Maier, S.F., Johnson, K.W., Leinwand, L.A., Chavez, R.A., Watkins, L.R. (2007). Intrathecal interleukin-10 gene therapy attenuates paclitaxel-induced mechanical allodynia and proinflammatory cytokine expression in dorsal root ganglia in rats. Brain Behav Immun 21, 686-698.

Li, Y., Zhang, H., Zhang, H., Kosturakis, A.K., Jawad, A.B., Dougherty, P.M. (2014). Toll-like receptor 4 signaling contributes to paclitaxelinduced peripheral neuropathy. J Pain 15, 712-725.

Materazzi, S., Fusi, C., Benemei, S., Pedretti, P., Patacchini, R., Nilius, B., Prenen, J., Creminon, C., Geppetti, P., Nassini, R. (2012). TRPAl and TRPV4 mediate paclitaxel-induced peripheral neuropathy in mice via a glutathione-sensitive mechanism. Pflugers Arch 463, 561569.

Mohan, M.J., Seaton, T., Mitchell, J., Howe, A., Blackburn, K., Burkhart, W., Moyer, M., Patel, I., Waitt, G.M., Becherer, J.D., Moss, M.L., Milla, M.E. (2002). The tumor necrosis factor-alpha converting enzyme (TACE): A unique metalloproteinase with highly defined substrate selectivity. Biochemistry 41, 9462-9469.

Peng, X.M., Zhou, Z.G., Glorioso, J.C., Fink, D.J., Mata, M. (2006). Tumor necrosis factor-alpha contributes to below-level neuropathic pain after spinal cord injury. Ann Neurol 59, 843-851.

Pittman, S.K., Gracias, N.G., Vasko, M.R., Fehrenbacher, J.C. (2014). Paclitaxel alters the evoked release of calcitonin gene-related peptide from rat sensory neurons in culture. Exp Neurol 253, 146153.

Sacerdote, P., Franchi, S., Trovato, A.E., Valsecchi, A.E., Panerai, A.E., Colleoni, M. (2008). Transient early expression of TNF-alpha in sciatic nerve and dorsal root ganglia in a mouse model of painful peripheral neuropathy. Neurosci Lett 436, 210-213.

Schafers, M., Geis, C., Svensson, C.I., Luo, Z.D., Sommer, C. (2003). Selective increase of tumour necrosis factor-alpha in injured and spared myelinated primary afferents after chronic constrictive injury of rat sciatic nerve. Eur J Neurosci 17, 791-804. 
Ustinova, E.E., Shurin, G.V., Gutkin, D.W., Shurin, M.R. (2013). The role of TLR4 in the paclitaxel effects on neuronal growth in vitro. PLOS ONE 8, e56886.

Wu, F.X., Bian, J.J., Miao, X.R., Huang, S.D., Xu, X.W., Gong, D.J., Sun, Y.M., Lu, Z.J., Yu, W.F. (2010). Intrathecal siRNA against Toll-like receptor 4 reduces nociception in a rat model of neuropathic pain. Int $J$ Med Sci 7, 251-259.

Wu, Z., Mata, M., Fink, D.J. (2011). Prevention of diabetic neuropathy by regulatable expression of HSV-mediated erythropoietin. Mol Ther 19, 310-317.

Wu, Z.Z., Chen, S.R., Pan, H.L. (2005). Transient receptor potential vanilloid type 1 activation down-regulates voltage-gated calcium channels through calcium-dependent calcineurin in sensory neurons. J Biol Chem 280, 18142-18151.

Yamakawa, I., Kojima, H., Terashima, T., Katagi, M., Oi, J., Urabe, H., Sanada, M., Kawai, H., Chan, L., Yasuda, H., Maegawa, H., Kimura, H (2011). Inactivation of TNF-alpha ameliorates diabetic neuropathy in mice. Am J Physiol Endocrinol Metab 301, E844-E852.

Zhou, Z., Peng, X., Hagshenas, J., Insolera, R., Fink, D.J., Mata, M. (2010). A novel cell-cell signaling by microglial transmembrane TNF alpha with implications for neuropathic pain. Pain 151, 296-306.

\section{Supporting Information}

Additional Supporting Information may be found in the online version of this article at the publisher's web-site:
Figure S1. In primary DRG neurons alone, direct exposure to paclitaxel did not induce the release of TNF- $\alpha$ or increase the expression of TRPAl or TRPV4. Primary DRG neurons in culture were treated with paclitaxel of different concentrations in the presence or the absence of TLR-4 inhibitor TAK242. TNF- $\alpha$ concentration in medium, and the protein levels of TRPAl and TRPV4 in cells were determined after $24 \mathrm{~h}$ of culture. (a) Release of TNF- $\alpha$ measured by ELISA. (b) Western blot of TRPAl and TRPV4.

Table S1. Primers used for gPCR.

Methods S1. Primary satellite glial cell culture.

Methods S2. Mechanical allodynia.

Methods S3. Cold allodynia.

Methods S4. Western blot.

Methods S5. Enzyme-linked immunosorbent assays (ELISA) for soluble TNF- $\alpha$ in medium and in DRG lysates, and TNFsR in medium.

Methods S6. TACE activity.

Methods S7. Immunohistochemistry.

Methods S8. Digoxigenin-labelled TNF- $\alpha$ RNA probe.

Methods S9. In situ hybridization of TNF- $\alpha$ mRNA. 